INPLASY PROTOCOL

To cite: Yan et al. Efficacy and safety of Chinese herbal medicine Xiao-qing-long-tang for allergic rhinitis: $A$ systematic review and metaanalysis of randomized controlled trials. Inplasy protocol 202160013. doi: 10.37766/inplasy2021.6.0013

Received: 06 June 2021

Published: 06 June 2021

Corresponding author: Yajie Yan

yyjay88@163.com

Author Affiliation: Guangzhou University of Chinese Medicine

Support: NSF(No.81974581).

Review Stage at time of this submission: Preliminary searches.

Conflicts of interest: None declared.

\section{Efficacy and safety of Chinese herbal medicine Xiao-qing-long-tang for allergic rhinitis: A systematic review and meta-analysis of randomized controlled trials}

\author{
Yan, Y1; Zhou, S2; Ruan, Y3; Li, Y4; Luo, Q5.
}

Review question / Objective: The efficacy and safety of Xiaoqing-long-Tang (XQLT) for allergic rhinitis.

Condition being studied: AR (Allergic rhinitis, AR) is an inflammatory disease of the nasal mucosa mediated by specific immunoglobulin E (IgE) and has become a global health problem. AR is mainly treated with nasal corticosteroids, antihistamines, and leukotriene receptor antagonists. However, these drugs can only provide temporary relief, and symptoms will recur soon after the drug is stopped. XQLT is a Classical prescription and originated from a book titled Shang han lun and is widely used for the treatment of pulmonary disorders, including AR and Asthma. There have been few clinical trials of XQLT for AR treatment. Our previous study also confirmed that XQLT can relieve the symptoms of AR patients and improve their quality of life. XQLT has pharmacological effects like an antihistamine, antiinflammatory, restored the Th1/Th2 and Th17/Treg cells balance. However, the efficacy and safety evidence of XQLT for $A R$ is still weak and remains to be verified.

INPLASY registration number: This protocol was registered with the International Platform of Registered Systematic Review and Meta-Analysis Protocols (INPLASY) on 06 June 2021 and was last updated on 06 June 2021 (registration number INPLASY202160013).

\section{INTRODUCTION}

Review question / Objective: The efficacy and safety of Xiao-qing-long-Tang (XQLT) for allergic rhinitis.

Condition being studied: AR (Allergic rhinitis, AR) is an inflammatory disease of the nasal mucosa mediated by specific immunoglobulin $E$ (IgE) and has become a global health problem. AR is mainly treated with nasal corticosteroids, antihistamines, and leukotriene receptor antagonists. However, these drugs can only provide temporary relief, and symptoms will recur soon after the drug is stopped. XQLT is a Classical prescription and originated from a book titled Shang han lun and is widely 
used for the treatment of pulmonary disorders, including AR and Asthma. There have been few clinical trials of XQLT for AR treatment. Our previous study also confirmed that XQLT can relieve the symptoms of AR patients and improve their quality of life. XQLT has pharmacological effects like an antihistamine, antiinflammatory, restored the Th1/Th2 and Th17/Treg cells balance. However, the efficacy and safety evidence of XQLT for $A R$ is still weak and remains to be verified.

\section{METHODS}

Participant or population: Patients had typical symptoms of allergic rhinitis (AR) and must be diagnosed by objective tests like a skin prick test or a serum IgE test. Participants of all ages, both genders with perennial allergic rhinitis (PAR) or seasonal allergic rhinitis (SAR) without classification limited were included. However, AR merged with other allergy diseases was excluded.

Intervention: All forms of oral administration of Xiao-qing-long-tang/SoCheong-Ryoung-Tang such as decoction, granule, capsule, tablet, or pill, used in comparison to placebo or conventional Western medicine were included for analysis. If XQLT was used together with other Chinese herbal medicine, only the trials using XQLT as the major formula with other herbs as the additional modification was involved. The dosage of Chinese herbs in XQLT accorded with the standard described in the 2020 edition of China Pharmacopoeia.

Comparator: We included studies that comparators only used any conventional treatment or placebo.

Study designs to be included: Only XQLT randomized controlled trials (RCTs) were included.

Eligibility criteria: Patients had typical symptoms of allergic rhinitis (AR) and must be diagnosed by objective tests like a skin prick test or a serum IgE test. Participants of all ages, both genders with perennial allergic rhinitis (PAR) or seasonal allergic rhinitis (SAR) without classification limited were included. However, AR merged with other allergy diseases was excluded.

Information sources: Cochrane Central Register of Controlled Trials (CENTRAL), PubMed, EMBASE (Excerpta Medical Database) and Clinical Trials.gov will be searched. We will also search the Chinese databases are China Biology Medicine databases (CBM), China National Knowledge Infrastructure (CNKI), Chinese Scientific Journals Full Text Database (CQVIP), Wanfang Data knowledge service platform (WanFang Data) and Chinese Clinical Trials Registry.

Main outcome(s): Any outcome recommended by any allergic rhinitis clinical handbook will be analysed. The primary outcome measures being total nasal symptom score (TNSS) or individual nasal symptom scores. One of the following outcomes was reported: TNSS (total nasal symptom scores, TNSS), individual symptom scores (rhinorrhea, nasal congestion, sneezing, and nasal itching), QoL (quality-of-life questionnaires, QoL) RQLQ (Rhinoconjunctivitis Quality of Life Questionnaire, RQLQ), VAS (Visual Analogue Scale, VAS), SF-36 (36-Item Short-Form Health Survey, SF-36), effective rate, the recurrence rate in the follow-up phase, sIgE (serum specific immunoglobulin E, slgE), IL-4 (serum interleukin 4, IL-4) level, and AEs (adverse events, AEs).

Quality assessment / Risk of bias analysis: Two researchers performed bias assessments using Cochrane collaboration's tool in seven domains: random sequence generation (selection bias), allocation concealment (selection bias), blinding of participants and personnel (performance bias), blinding of outcome assessment (detection bias), incomplete outcome data (attrition bias), selective reporting (reporting bias), and other bias and classified items as "low risk," "high risk," or "unclear risk". All reviewers resolved any conflicts by discussion. 
Strategy of data synthesis: The Cochrane systematic review software Review Manager 5.4.1 and Stata software are used for performing analysis and statistics. Review Manager 5.4.1were used to draw forest plots, perform subgroup analyses, evaluate risks of bias, and determine publication bias. Stata 14.0 software applied to perform sensitivity analysis. Variable data like total nasal symptom scores, MD (mean difference, MD) was used when the outcomes were measured with the same scale. When various scales were be utilized for outcome measures, SMD (standardized mean difference, SMD) was used. The effect rate and recurrence rate, which belong to dichotomous data, OR (Odds ratio, OR) was used to analyze inverse variance. Overall results were presented with $95 \% \mathrm{Cl}$ (confidence interval, $\mathrm{Cl})$. Heterogeneity was presented with the Cochrane $Q$ statistic and the 12 statistic. A fixed-effects model was applied to measure when the 12 value was $<50 \%$ and the Cochrane $Q$ p-value $>0.10$, indicated non-significant heterogeneity across studies. Otherwise, a random-effects model was employed. Publication bias is evaluated by a funnel plot.

Subgroup analysis: Specific subgroups were analyzed according to analogous intervention forms or design.

Sensitivity analysis: (1) The effect of risk of bias in included studies-defined as adequate allocation concealment and blinding of outcomeassessors; (2) the effect of using XQLT supplements.

Language: English.

Country(ies) involved: China.

Keywords: Allergic rhinitis; Xiao-qing-longtang; RCT.

Contributions of each author:

Author 1 - Yajie Yan - The author examined the search results for extracting information and drafted the manuscript.

Email: yyjay88@163.com
Author 2 - Shiqing Zhou - The author designed and carried out the search strategy.

Email: shiqingzhou@gzucm.edu.cn

Author 3 - Yan Ruan - The author contributed to the development of the selection criteria, and the risk of bias assessment strategy.

Email: ry0722@gzucm.edu.cn

Author 4 - Yunying Li - The author read, provided feedback and approved the final manuscript.

Email: gdliyunying@163.com

Author 5 - Qiulan Luo - The author provided statistical expertise.

Email: 13760713695@126.com 